\title{
Strategic Abstention based on Preference Extensions: Positive Results and Computer-Generated Impossibilities
}

\author{
Florian Brandl \\ Stanford University \\ Palo Alto, USA \\ Felix Brandt \\ Christian Geist \\ Johannes Hofbauer \\ Technical University of Munich \\ Munich, Germany
}

BRANDL.FFX@GMAIL.COM

\author{
BRANDTF@IN.TUM.DE \\ GEIST@IN.TUM.DE \\ HOFBAUEJ@IN.TUM.DE
}

\begin{abstract}
Voting rules allow multiple agents to aggregate their preferences in order to reach joint decisions. A common flaw of some voting rules, known as the no-show paradox, is that agents may obtain a more preferred outcome by abstaining from an election. We study strategic abstention for set-valued voting rules based on Kelly's and Fishburn's preference extensions. Our contribution is twofold. First, we show that, whenever there are at least five alternatives and seven agents, every Pareto-optimal majoritarian voting rule suffers from the no-show paradox with respect to Fishburn's extension. This is achieved by reducing the statement to a finite - yet very large - problem, which is encoded as a formula in propositional logic and then shown to be unsatisfiable by a SAT solver. We also provide a human-readable proof which we extracted from a minimal unsatisfiable core of the formula. Secondly, we prove that every voting rule that satisfies two natural conditions cannot be manipulated by strategic abstention with respect to Kelly's extension and give examples of well-known Pareto-optimal majoritarian voting rules that meet these requirements.
\end{abstract}

\section{Introduction}

Whenever a group of multiple agents aims at reaching a joint decision in a fair and satisfactory way, they need to aggregate their (possibly conflicting) preferences. Voting rules are studied in detail in social choice theory and are coming under increasing scrutiny from computer scientists who are interested in their computational properties or want to utilize them in artificial multiagent systems (Chevaleyre, Endriss, Lang, \& Maudet, 2007; Brandt, Conitzer, \& Endriss, 2013; Brandt, Conitzer, Endriss, Lang, \& Procaccia, 2016; Endriss, 2017).

A common flaw of many such rules, first observed by Fishburn and Brams (1983) is that agents may obtain a more preferred outcome by abstaining from an election. This phenomenon is known as the no-show paradox. Following Moulin (1988), a voting rule is said to satisfy participation if it is immune to the no-show paradox. Moulin has shown that all resolute, i.e., single-valued, scoring rules (such as Borda's rule) satisfy participation while all resolute Condorcet extensions suffer from the no-show paradox. Condorcet extensions comprise a large class of voting rules that satisfy otherwise rather desirable properties. 
In this paper, we study participation for irresolute, i.e., set-valued, social choice functions (SCFs). A proper definition of participation for irresolute SCFs requires the specification of preferences over sets of alternatives. Rather than asking the agents to specify their complete preferences over all subsets (which would be bound to various rationality constraints and require exponential space), it is typically assumed that the preferences over single alternatives can be extended to preferences over sets. Of course, there are various ways how to extend preferences to sets (see, e.g., Gärdenfors, 1979; Barberà, Bossert, \& Pattanaik, 2004), each of which leads to a different version of participation. A function that yields a (possibly incomplete) preference relation over subsets of alternatives when given a preference relation over single alternatives is called a preference extension. In this paper, we focus on two common preference extensions due to Kelly (1977) and Fishburn (1972), both of which arise under natural assumptions about the agents' knowledge of the tie-breaking mechanism that eventually picks a single alternative from the choice set (see, e.g., Gärdenfors, 1979; Ching \& Zhou, 2002; Sanver \& Zwicker, 2012; Brandt \& Brill, 2011; Brandt, 2015; Brandt, Saile, \& Stricker, 2018).

Kelly's extension, for example, can be motivated by assuming that the agents are completely unaware of how ties are broken. A common interpretation of Fishburn's extension, on the other hand, is that ties are broken according to the unknown but transitive preferences of a chairman. While the agents do not know the chairman's preferences, they are aware that tie-breaking is consistent across different sets. Since Fishburn's extension is a refinement of Kelly's extension it follows that Fishburn-participation is stronger than Kellyparticipation. In this paper we exploit the uncertainty of the agents about the tie-breaking mechanism in order to prevent strategic abstention. Our two main results are as follows.

- Whenever there are at least four alternatives and six agents, Pareto-optimality and Fishburn-participation are incompatible in the context of majoritarian SCFs. When there are at least five alternatives and seven agents, this even holds for strict preferences. These results imply impossibilities for SCFs with uniformly randomized tiebreaking, even when the agents' concrete utility functions are unknown.

- Every SCF that satisfies set-monotonicity and is independent of indifferent voters satisfies Kelly-participation. Every set-monotonic majoritarian SCF satisfies Kellyparticipation when preferences are strict.

The first result is obtained using computer-aided theorem proving techniques. In particular, we reduce the statement to a finite - yet very large - problem, which is encoded as a formula in propositional logic and then shown to be unsatisfiable by a SAT solver. We also provide a human-readable proof for this result, which we extracted from a minimal unsatisfiable core of the SAT formula.

The conditions for the second result are easy to check and satisfied by a small number of well-studied SCFs, including Pareto-optimal majoritarian SCFs. In contrast to Moulin's negative result for resolute SCFs, there are appealing Condorcet extensions that satisfy Kelly-participation.

Our negative result holds even for strict preferences while our positive result holds even for weak preferences. The latter is somewhat surprising and stands in sharp contrast to the related finding that no Condorcet extension satisfies Kelly-strategyproofness when 
preferences are weak (recall that an SCF is strategyproof if no agent can obtain a more preferred outcome by misrepresenting his preferences) (Brandt, 2015).

Participation is similar to, but logically independent from, strategyproofness. Manipulation by abstention is arguably a more severe problem than manipulation by misrepresentation for two reasons. First, agents might not be able to find a beneficial misrepresentation. Various authors have shown that the corresponding computational problem can be intractable (see, e.g., Faliszewski, Hemaspaandra, \& Hemaspaandra, 2010). Finding a successful manipulation by strategic abstention, on the other hand, is never harder than computing the outcome of the SCF. Secondly, one could argue that agents will not lie about their preferences because they consider it to be immoral (Borda famously exclaimed "my scheme is intended only for honest men"), while they view strategic abstention as acceptable. $^{1}$

The remainder of the paper is structured as follows: we provide an overview of related work in Section 2 and introduce the theoretical foundations and the notions of participation in Section 3. Section 4 gives a short introduction to computer-aided theorem proving and focuses on encoding participation. The results obtained are presented and discussed in Section 5 and briefly summarized in Section 6 . For the proofs we originally found with the help of a computer, we provide human-readable versions that we manually restructured to improve readability. The tedious proof of our theorem for strict preferences can be found in Section 6.

\section{Related Work}

The problem of strategic abstention for irresolute SCFs has been addressed by a number of authors. Pérez (2001) examined the situation where an agent can cause his most preferred alternative to be excluded from the choice set when joining an electorate and showed that almost all common Condorcet extensions suffer from this paradox. Jimeno, Pérez, and García (2009) proved that manipulation by abstention is possible for most common Condorcet extensions when agents compare sets according to an optimistic, pessimistic, or lexicographic preference extension. As observed by Brandt, Geist, and Peters (2017), who also complemented these results with tight bounds on the number of required agents, the impossibility for optimistic agents already follows from Moulin's (1988) theorem. Jimeno et al. mentioned the investigation of participation for weak preferences and for Fishburn's extension as interesting research directions for future work. Both of these questions are addressed in our paper. Pérez, Jimeno, and García (2010) and Pérez, Jimeno, and García (2015) revisited participation for the optimist extension with varying notions of Condorcet-consistency. Pérez et al. (2010) derived incompatibilities using a set-valued variant of Condorcet-consistency for irresolute SCFs that always select exactly $k$ alternatives. Inspired by early work by Holzman (1988) on resolute SCFs, Pérez et al. (2015) showed that participation is compatible with Condorcet-consistency if Condorcet winners need the support of a supermajority rather than a simple majority. Brandt (2015) mainly focused on strategyproofness for Kelly's extension but in Remark 9 also gave a simple argument connecting strategyproofness and participation.

1. Alternatively, one could also argue that manipulation by misrepresentation is more critical because agents are tempted to act immorally, which is a valid, but different, concern. 
The computer-aided techniques in this paper have been inspired by Tang and Lin (2009), who reduced well-known impossibility results to finite instances, which can then be checked by a SAT solver. Geist and Endriss (2011) extended this method to a fully-automatic search algorithm for impossibility theorems in the context of preference relations over sets of alternatives. More recently, Brandt and Geist (2016) proved both impossibility and possibility results regarding the strategyproofness of irresolute SCFs using this computeraided approach. We strongly build on their methodology and extend it to cover the notion of participation, which - as we will see - requires a more advanced framework. Brandt et al. (2017) used SAT solving together with participation in a different context: they considered Moulin's original result (Moulin, 1988) - and variants thereof - and examined the minimal number of agents needed for the no-show paradox to occur.

\section{Preliminaries}

Let $A$ be a finite set of alternatives and $\mathbb{N}$ a set of agents of which we will consider finite subsets $N \subseteq \mathbb{N}$. Therefore, let $\mathcal{F}(\mathbb{N})$ denote the set of all finite and non-empty subsets of $\mathbb{N}$. A (weak) preference relation is a complete, reflexive, and transitive binary relation on $A$. The preference relation of agent $i$ is denoted by $\succsim_{i}$. The set of all preference relations is denoted by $\mathcal{R}$. We write $\succ_{i}$ for the strict part of $\succsim_{i}$, i.e., $x \succ_{i} y$ if $x \succsim_{i} y$ but not $y \succsim_{i} x$, and $\sim_{i}$ for the indifference part of $\succsim_{i}$, i.e., $x \sim_{i} y$ if $x \succsim_{i} y$ and $y \succsim_{i} x$. A preference relation $\succsim_{i}$ is called strict if it additionally is anti-symmetric, i.e., $x \succ_{i} y$ or $y \succ_{i} x$ for all distinct alternatives $x, y$. We will compactly represent a preference relation as a comma-separated list with all alternatives among which an agent is indifferent placed in a set. For example $x \succ_{i} y \sim_{i} z$ is represented by $\succsim_{i}: x,\{y, z\}$.

A preference profile $R$ is a function from a set of agents $N$ to the set of preference relations $\mathcal{R}$. The set of all preference profiles is denoted by $\mathcal{R}^{\mathcal{F}(\mathbb{N})}$. For a preference profile $R \in \mathcal{R}^{N}$ and two agents $i \in N, j \in \mathbb{N}$, we define

$$
R_{-i}=R \backslash\left\{\left(i, \succsim_{i}\right)\right\} \quad \text { and } \quad R_{+j}=R \cup\left\{\left(j, \succsim_{j}\right)\right\} .
$$

When multiple agents join an electorate, we sometimes also use $R_{+j_{1}, \ldots, j_{k}}=R \cup\left(\bigcup_{1 \leq l \leq k}\left(j_{l}, \succsim_{j_{l}}\right)\right)$.

For a preference profile $R$ and two alternatives $x, y$, the majority margin of $x$ over $y$, $w_{R}(x, y)=\left|\left\{i \in N: x \succsim_{i} y\right\}\right|-\left|\left\{i \in N: y \succsim_{i} x\right\}\right|$, is the number of agents who prefer $x$ to $y$ minus the number of agents who prefer $y$ to $x$. We will omit $R$ whenever it is clear from the context. The majority relation of $R$ is $\succsim_{R}$, where $x \succsim_{R} y$ iff $w_{R}(x, y)>0$. We denote its strict part by $\succ_{R}$ and its indifference part by $\sim_{R}$. An alternative $x$ is a Condorcet winner in $R$ if $x \succ_{R} y$ for all $y \in A \backslash\{x\}$. Figure 1 illustrates the preceding definitions.

For our proofs we will frequently need preference profiles that induce particular majority margins. The following result by Debord (1987) shows that such profiles exist. It also implies McGarvey's (1953) theorem, which shows that every tournament is induced by some preference profile.

Proposition 1 (Debord, 1987). Let $W \in \mathbb{N}^{|A| \times|A|}$ be a skew-symmetric matrix with either only even or only odd off-diagonal entries. ${ }^{2}$ Then there is a preference profile $R$ such that $w_{R}(x, y)=W(x, y)$ for all alternatives $x, y$.

2. A matrix $W$ is skew-symmetric if $W=-W^{T}$. 


\begin{tabular}{lll}
1 & 2 & 3 \\
\hline$a$ & $c$ & $d$ \\
$b$ & $a$ & $b$ \\
$c$ & $d$ & $a$ \\
$d$ & $b$ & $c$ \\
& $R$ &
\end{tabular}

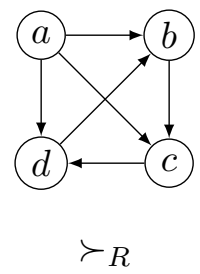

Figure 1: $R$ is a preference profile on four alternatives and three agents. The numbers in the top row denote the agents' identities. Each column states the preferences of one agent, e.g., agent 1 prefers $a$ to $b$ to $c$ to $d$. The directed graph on the right depicts the strict part of the majority relation $\succsim_{R}$ of $R$. There is an edge from $x$ to $y$ if a majority of agents prefers $x$ to $y$, e.g., agents 1 and 2 prefer $a$ to $b$, so $a \succ_{R} b$ and there is an edge from $a$ to $b$. Moreover, $a$ is a Condorcet winner.

Our central objects of study are social choice functions (SCFs), i.e., functions that map a preference profile to a set of alternatives. Formally, an SCF is a function

$$
f: \mathcal{R}^{\mathcal{F}(\mathbb{N})} \rightarrow 2^{A} \backslash \emptyset .
$$

Two minimal fairness conditions for SCFs are anonymity and neutrality. An SCF is anonymous if the outcome does not depend on the identities of the agents and neutral if it is symmetric with respect to alternatives. More formally, $f$ is anonymous if $f(R)=f\left(R^{\prime}\right)$ for all $R \in \mathcal{R}^{N}, R^{\prime} \in \mathcal{R}^{N^{\prime}}, N, N^{\prime} \in \mathcal{F}(\mathbb{N})$ and all bijections $\pi: N \rightarrow N^{\prime}$ such that $\left(\succsim_{i}\right)_{i \in N}=\left(\succsim^{\prime}{ }_{\pi(i)}\right)_{i \in N}$. To define neutrality, denote by $\succsim_{i}{ }^{\pi}$ the result of renaming alternatives in the preference relation $\succsim_{i}$ according to the permutation $\pi$ of $A$, i.e., $\pi(x) \succsim_{i}^{\pi} \pi(y)$ if and only if $x \succsim_{i} y$. Then we get $R^{\pi}=\left(\succsim_{i}{ }^{\pi}\right)_{i \in N}$ by renaming alternatives in each agent's preference relation. We say that $f$ is neutral if $f\left(R^{\pi}\right)=\pi(f(R))$ for all $N \in \mathcal{F}(\mathbb{N})$ and all permutations $\pi: A \rightarrow A$.

An SCF $f$ is majoritarian (or a neutral $\mathrm{C} 1$ function) if it is neutral and for all $R, R^{\prime} \in \mathcal{R}^{\mathcal{F}(\mathbb{N})}, f(R)=f\left(R^{\prime}\right)$ whenever $\succ_{R}=\succ_{R^{\prime}}$. Even the seemingly narrow class of majoritarian SCFs contains a variety of interesting functions (sometimes called tournament solutions) such as

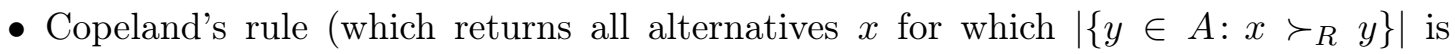
maximal),

- the top cycle (which returns the maximal elements of the transitive closure of $\succsim_{R}$ ),

- the uncovered set (which returns the maximal elements of a subrelation of $\succsim_{R}$ known as the covering relation, see Section 5.1), and

- the bipartisan set (which returns all alternatives that are played with positive probability in some mixed-strategy equilibrium of the zero-sum game $\left.\left(\operatorname{sgn}\left(w_{R}(x, y)\right)\right)_{x, y \in A}\right)$.

All of the mentioned SCFs happen to be Condorcet extensions or Condorcet-consistent $S C F s$, i.e., SCFs that uniquely return a Condorcet winner whenever one exists. We refer to 
Laslier (1997) and Brandt, Brill, and Harrenstein (2016) for a much more detailed overview of majoritarian SCFs. In particular, the bipartisan set turned out to satisfy a large number of desirable properties.

Next we introduce a very weak variable electorate condition which requires that a completely indifferent agent does not affect the outcome. An SCF $f$ satisfies independence of indifferent voters (IIV) if

$$
f(R)=f\left(R_{+i}\right) \text { for all } R \in \mathcal{R}^{\mathcal{F}(\mathbb{N})} \text { whenever } x \sim_{i} y \text { for all } x, y \in A,
$$

i.e., adding an agent who is indifferent between all alternatives does not change the outcome. It is easy to see that every majoritarian SCF satisfies anonymity, neutrality, and IIV.

Set-monotonicity. We say that $R^{\prime}$ is an $f$-improvement over $R$ if alternatives that are chosen by $f$ in $R$ are not weakened from $R$ to $R^{\prime}$, i.e., for all $x \in f(R), y \in A$, and $i \in N$, $x \succsim_{i} y$ implies $x \succsim_{i}^{\prime} y$ and $y \succsim_{i}^{\prime} x$ implies $y \succsim_{i} x$. An SCF $f$ satisfies set-monotonicity if

$$
f(R)=f\left(R^{\prime}\right) \text { whenever } R^{\prime} \text { is an } f \text {-improvement over } R \text {. }
$$

Set-monotonicity was introduced by Brandt (2015) in the context of Kellystrategyproofness.

Preference extensions. The two preference extensions we consider in this paper are Kelly's extension and Fishburn's extension. For all $X, Y \subseteq A$ and $\succsim_{i} \in \mathcal{R}$,

$$
\begin{aligned}
& X \succsim_{i}^{K} Y \text { iff } x \succsim_{i} y \text { for all } x \in X, y \in Y, \text { and } \\
& X \succsim_{i}^{F} Y \text { iff } X \backslash Y \succsim_{i}^{K} Y \text { and } X \succsim_{i}^{K} Y \backslash X .
\end{aligned}
$$

The strict part of these relations will be denoted by $\succ_{i}^{K}$ and $\succ_{i}^{F}$, respectively. It follows from the definitions that Fishburn's extension is a refinement of Kelly's extension. Hence, $\succsim_{i}^{K} \subseteq \succsim_{i}^{F}$ for every $\succsim_{i} \in \mathcal{R}$, i.e., whenever one set is Kelly-preferred to another one, it is also Fishburn-preferred.

For better illustration of these extensions consider the preference relation $\succsim_{i}: a, b, c, d$ and the sets of alternatives $X=\{a, b\}, Y=\{a, b, c\}$, and $Z=\{b, d\}$. Then, $X \succ_{i}^{F} Y$ and $X \succ_{i}^{K} Z$. Sets $Y$ and $Z$ are not comparable with respect to either one of the two extensions.

Both extensions can be motivated using randomized tie-breaking mechanisms and expected utility maximization. Assume it is known that the tie-breaking lottery puts positive probabilities on all alternatives in the choice set (and no other alternatives), but the exact probabilities as well as the underlying utility function are unknown. ${ }^{3}$ Then Kelly's extension is obtained by postulating that one set is preferred to another if the former yields at least as much expected utility as the latter for all lotteries whose support coincides with the respective choice set and all consistent utility functions. For Fishburn's extension, one may additionally assume that an unknown positive weight is assigned to every alternative $a$ priori and, given some choice set, the tie-breaking lottery over this set has probabilities directly proportional to these weights. The most natural special case is a weight function that assigns equal weights to all alternative, which results in uniform lotteries over alternatives in any choice set.

3. Utility functions are required to be consistent with the ordinal preferences, i.e., if $x \succsim_{i} y$, then $x$ has to yield at least as much utility as $y$. 
Participation and strategyproofness. With the preference extensions at hand, we can now formally define participation and strategyproofness. An SCF $f$ is Kelly-manipulable by strategic abstention if there exists a preference profile $R \in \mathcal{R}^{N}$ with $N \in \mathcal{F}(\mathbb{N})$ and an agent $i \in N$ such that $f\left(R_{-i}\right) \succ_{i}^{K} f(R)$. An SCF $f$ is Kelly-manipulable if there exist preference profiles $R, R^{\prime} \in \mathcal{R}^{N}$, and an agent $i \in N$, such that $\succsim_{j}=\succsim_{j}^{\prime}$ for all agents $j \neq i$ and $f\left(R^{\prime}\right) \succ_{i}^{K} f(R)$. $f$ satisfies Kelly-participation or Kelly-strategyproofness if it is not Kelly-manipulable by strategic abstention or Kelly-manipulable, respectively. Fishburnparticipation and Fishburn-strategyproofness are defined analogously. The interpretation of Fishburn's extension above shows that an impossibility for Fishburn-participation implies an impossibility for SCFs with randomized tie-breaking using uniform lotteries (and unknown utility functions).

The following example illustrates the definitions of Kelly-participation and Fishburnparticipation. Consider the preference profile $R$ with six agents and four alternatives depicted below. Note that agents 3 and 4 as well as agents 5 and 6 have identical preferences.

\begin{tabular}{cccc}
1 & 2 & 3,4 & 5,6 \\
\hline$c$ & $d$ & $a$ & $b$ \\
$d$ & $b$ & $c$ & $a$ \\
$b$ & $a$ & $d$ & $c$ \\
$a$ & $c$ & $b$ & $d$ \\
& \multicolumn{2}{c}{$R$} &
\end{tabular}
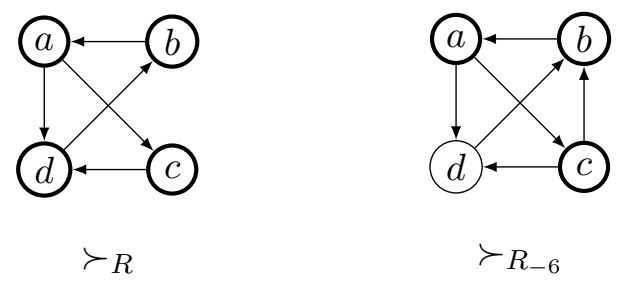

The profile $R$ induces the majority relation $\succsim_{R}$ with its strict part $\succ_{R}$. The bipartisan set of $R$ can be computed to be $\{a, b, c, d\} .{ }^{4}$ If agent 6 leaves the electorate, we obtain the profile $R_{-6}$, which induces the majority relation $\succsim_{R_{-6}}$ whose bipartisan set is $\{a, b, c\}$. Observe that $\{a, b, c\} \succ_{6}^{F}\{a, b, c, d\}$, i.e., agent 6 can obtain a preferred outcome according to Fishburn's extension by abstaining from the election. Hence, the bipartisan set does not satisfy Fishburn-participation. However, $\{a, b, c\} \succ_{6}^{K}\{a, b, c, d\}$ does not hold and, hence, agent 6 cannot manipulate by abstaining according to Kelly's extension. This is not a coincidence: the bipartisan set satisfies Kelly-participation because it satisfies set-monotonicity and IIV (cf. Theorem 3).

Pareto-optimality. In the subsequent sections, we will use participation and strategyproofness in conjunction with Pareto-optimality. An alternative $x$ is said to be Paretodominated (in $R \in \mathcal{R}^{N}$ ) by another alternative $y$ if $y \succsim_{i} x$ for all $i \in N$ and there exists $j \in N$ such that $y \succ_{j} x$. Whenever there is no $y \in A$ that Pareto-dominates $x, x$ is Pareto-optimal. An SCF is Pareto-optimal if it never selects a Pareto-dominated alternative. The Pareto rule $(P O)$ is defined as the $\mathrm{SCF}$ that returns all Pareto-optimal alternatives.

\section{Computer-Aided Theorem Proving}

For our impossibility theorems, we will make use of the computer-aided proving methodology described by Brandt and Geist (2016). The main idea is to prove statements by encoding a finite instance as a satisfiability problem, which can be solved by a computer

4. Computing the bipartisan set is non-trivial - but feasible in polynomial time - because it requires solving a linear program. In the given example, the bipartisan set coincides with the uncovered set. 


\begin{tabular}{ccc}
\hline$|A|$ & Brandt and Geist (2016) & This paper \\
\hline 3 & 49 & $\mathbf{8 2 3 , 5 4 3}$ \\
4 & 50,625 & $\sim \mathbf{2 . 5} \cdot \mathbf{1 0}^{\mathbf{4 9}}$ \\
5 & $\sim 7.9 \cdot 10^{17}$ & $\sim \mathbf{9 . 4} \cdot \mathbf{1 0}^{\mathbf{8 6 7}}$ \\
6 & $\sim 5.8 \cdot 10^{100}$ & $\sim \mathbf{6 . 8} \cdot \mathbf{1 0}^{\mathbf{3 8 6 4 9}}$ \\
\hline
\end{tabular}

Table 1: Number of different majoritarian SCFs for 3 to 6 alternatives. For every majority relation, an SCF can choose one out of $2^{|A|}-1$ possible sets of alternatives. So the number of SCFs is exponential in the number of different majority relations. While Brandt and Geist (2016) could assume an odd number of agents with strict preferences resulting in strict majority relations, participation requires us to deal with variable electorates, and therefore possibly even numbers of agents and majority relations that need not be strict.

using a SAT solver, and then providing a (simple) reduction argument which extends this result to arbitrary domain sizes. We extend their framework to also cater for indifferences in the majority relations, which is an important requirement for being able to deal with participation: if an agent with at least one strict preference abstains from the election, the corresponding majority relation might already contain indifferences.

Note that introducing majority ties significantly increases the size of the search space (see Table 1). Apart from being able to treat such large search spaces, another major advantage of the computer-aided approach is that many similar conjectures and hypotheses (e.g., statements using other preference extensions) can be checked quickly using the same framework.

In the following subsections, we are going to explain our extension and some core features of the computer-aided method; for details of the original approach, however, the reader is referred to Brandt and Geist (2016) and Geist and Peters (2017).

\subsection{Encoding Participation}

At the core of the computer-aided approach lies an encoding of the problem to be solved as a SAT instance. For this, all axioms involved need to be stated in propositional logic. We take over the formalization of the optimized encoding by Brandt and Geist (2016), which contains the following relevant axioms: functionality of the choice function, the orbit condition, and Pareto-optimality. Pareto-optimality is encoded as being a refinement of the uncovered set (see Section 5.1 for details). What remains is to encode the notion of participation. In order to be able to deal with participation, we introduce a new condition called majority-participation and prove that it is equivalent to participation for majoritarian SCFs.

We are going to identify preference profiles with their corresponding majority relations (i.e., for majoritarian SCFs $f$ we often also write $f\left(\succsim_{R}\right)$ instead of $\left.f(R)\right)$. Moreover, the inverse of $\succsim \in \mathcal{R}$ is denoted by $\precsim$, i.e., $x \precsim y$ if and only if $y \succsim x$. 
Definition 1. A majoritarian SCF $f$ is Fishburn-majority-manipulable by strategic abstention if there exist majority relations $\succsim, ~ \succsim^{\prime}$ and a preference relation $\succsim_{i} \in \mathcal{R}$ such that $f\left(\succsim^{\prime}\right) \succ_{i}^{F} f(\succsim)$, with

$$
\begin{aligned}
& \succ \cap \prec^{\prime}=\emptyset, \\
&\left(\succsim \backslash \succsim^{\prime}\right) \cup\left(\precsim^{\prime} \backslash \precsim\right) \subseteq \succ_{i}, \text { and } \\
& \sim \cap \sim^{\prime} \subseteq \sim_{i} .
\end{aligned}
$$

Whenever we require the agents' preferences to be strict, either $\succsim$ or $\succsim^{\prime}$ has to be antisymmetric in addition. A majoritarian SCF $f$ satisfies Fishburn-majority-participation if it is not Fishburn-majority-manipulable by strategic abstention.

Conditions (1) to (3) can be interpreted as follows: (1) prescribes that no strict relationship may be reversed between $\succ$ and $\succ^{\prime}$. (2) requires that $\succsim_{i}$ is in line with the changes from $\succsim$ to $\succsim^{\prime}$; more precisely, the first part of the set inclusion prescribes that a majority tie between two alternatives $x$ and $y$ in $\succsim$ can only turn into a strict preference of $x$ over $y$ in $\succsim^{\prime}$, if the abstaining agent $i$ strictly prefers $y$ to $x$; the second part requires the analogous statement for alternatives less preferred by agent $i$. (3) means that majority ties that occur in both majority relations must be reflected by an indifference in $\succsim_{i}$, so ties in the majority relation can only be broken if the abstaining agent $i$ is not indifferent himself.

In the following lemma, we show that, for majoritarian SCFs, the condition of Fishburnmajority-manipulability corresponds to an abstaining agent with preferences $\succsim_{i}$ who thereby obtains a preferred outcome. ${ }^{5}$

Lemma 1. A majoritarian SCF satisfies Fishburn-participation if and only if it satisfies Fishburn-majority-participation.

Proof. We first provide a short outline of the proof. To begin with, we show that for every preference profile $R$ that allows for a Fishburn-manipulation by abstention by agent $i$, the two majority relations $\succsim_{R}$ and $\succsim_{R_{-i}}$ together with $\succsim_{i}$ satisfy all required conditions. In return, whenever we have two majority relations $\succsim^{1}, \succsim^{2}$ and a preference relation $\succsim_{i}$, all with the properties stated in Definition 1, we can assign integer majority margins to all pairs of alternatives and, by Proposition 1, use these to determine a preference profile $R^{2}$ that induces the majority relation $\succsim^{2}$. Together with $R=R_{+i}^{2}$ we obtain $\succsim_{R^{2}}=\succsim^{2}, \succsim_{R}=\succsim^{1}$ and thus $f\left(R^{2}\right) \succ_{i}^{F} f(R)$ for any majoritarian SCF $f$.

In more detail, we begin by showing that if a majoritarian SCF violates Fishburnparticipation, then it also violates Fishburn-majority-participation. Therefore, let $f$ be a majoritarian SCF such that there exists some electorate $N$, agenda $A$, preference profile $R \in$ $\mathcal{R}^{N}$ and agent $i \in N$ for whom it holds that $f\left(R_{-i}\right) \succ_{i}^{F} f(R)$, i.e., there is an instance where $i$ can strategically manipulate by abstaining. Let furthermore $\succsim^{1}=\succsim_{R}$ and $\succsim^{2}=\succsim_{R_{-i}}$.

First note that we trivially have that $f\left(\succsim^{2}\right) \succ_{i}^{F} f\left(\succsim^{1}\right)$. Now, continue with conditions (1) to (3) as given in Definition 1. Since only one agent leaves the electorate when going from $R$ to $R_{-i}$ and everything else remains unchanged, we cannot have that $x \succ^{1} y$ and

5. Note that both the definition of majority-participation and Lemma 1 are independent of a specific preference extension, and thus also applicable to, e.g., Kelly's extension. 
$y \succ^{2} x$ for any $x, y \in A$, i.e., no edges in the strict part of the majority relation can be reversed. Hence, $\succ^{1} \cap \prec^{2}=\emptyset$.

Next, we have that if an alternative is preferred to another alternative by a majority in $R$ and not preferred any more in $R_{-i}$, than $i$ must prefer the former to the latter. Conversely, given one alternative is preferred to another alternative by a majority in $R_{-i}$ but not in $R$, $i$ has to prefer the latter to the former. We together obtain $\left(\succsim \backslash \succsim^{\prime}\right) \cup\left(\precsim^{\prime} \backslash \precsim\right) \subseteq \succ_{i}$.

Additionally, for every majority comparison that was balanced at an indifference in both $R$ and $R_{-i}$, we necessarily also have an indifference in $i$ 's preferences: $\sim^{1} \cap \sim^{2} \subseteq \sim_{i}$.

Finally note that either $|N|$ or $(|N|-1)$ is odd, so, assuming strict individual preferences, we trivially have that either $\succsim^{1}$ or $\succsim^{2}$ does not contain majority indifferences, i.e., is antisymmetric.

We continue with the reverse direction and show that if $f$ violates Fishburn-majorityparticipation, then it also violates Fishburn-participation. To this end, let $f$ be a majoritarian SCF, $\succsim^{1}$ and $\succsim^{2}$ be two majority relations (on some set of alternatives $A$ ), $\succsim_{i} \in \mathcal{R}$ an individual preference ranking such that $f\left(\succsim^{2}\right) \succ_{i}^{F} f\left(\succsim^{1}\right)$, and let (1) to (3) as given in Definition 1 hold. Concerning individual preferences, we start with the general case.

We begin with $\succsim^{2}$ and assign margins to all majority comparisons $(x, y) \in \succ^{2}$ such that

$$
w(x, y)= \begin{cases}1 & \text { if }(x, y) \notin \succ^{1}, \text { and } \\ 3 & \text { if }(x, y) \in \succ^{1} .\end{cases}
$$

Using Proposition 1, it is possible to construct a preference profile $R^{2}$ (for some electorate $\left.N^{2}\right)$ such that $\succsim_{R^{2}}=\succsim^{2}$ and $\left|\left\{j \in N: x \succsim_{j}^{2} y\right\}\right|-\left|\left\{j \in N: y \succsim_{j}^{2} x\right\}\right|=w(x, y)$ for all $x, y \in A$. Define $R=R_{+i}^{2}$ (and consequently $N=N^{2} \cup\{i\}$ ). We now show that $\succsim_{R}=\succsim^{1}$, or, equivalently, that all changes when going from $\succsim^{2}$ to $\succsim^{1}$ are solely due to $i$ joining the electorate while at the same time no other majority comparisons change.

Therefore note that for every pair of alternatives $x, y \in A$, one of the following cases - or its neutral equivalent-applies:

1. $(x, y) \in \succ^{1} \cap \succ^{2}$ : In this case $w(x, y)=3$ and $i$ 's preferences do not affect the majority comparison.

2. $(x, y) \in \succ^{1},(y, x) \in \succ^{2}$ : This case is not possible due to (1) in Definition 1.

3. $(x, y) \in \sim^{1},(x, y) \in \succ^{2}$ : We here have $w(x, y)=1$ and by (2) in Definition 1 we know that $y \succ_{i} x$, i.e., including $i$ indeed causes the majority comparison to change in the required way.

4. $(x, y) \in \succ^{1},(x, y) \in \sim^{2}$ : By (2) in Definition 1 we know that $x \succ_{i} y$ and hence adding $i$ obviously causes the majority indifference in $\succsim^{2}$ to sway in favor of $x$.

5. $(x, y) \in \sim^{1},(x, y) \in \sim^{2}$ : By (3) in Definition 1 this means that $x \sim_{i} y, i$ does not affect the majority comparison and it remains identical.

Consequently $\succsim_{R}=\succsim^{1}$ and we directly deduce that $f\left(R_{-i}\right) \succ_{i}^{F} f(R)$, i.e., based on a Fishburn-majority-manipulation we have constructed a Fishburn-manipulation and $f$ violates Fishburn-participation. 
In case the agents' preferences are required to be strict, minor details of the proof change. First note that by Definition 1, either $\succsim^{1}$ or $\succsim^{2}$ has to be anti-symmetric. If $\succsim^{1}$ is anti-symmetric, $\succsim^{2}$ is not and we have to slightly modify our margin function for $\succsim^{2}$ and instead use $w(x, y)=2$ for all $(x, y) \in \succ^{2}$. This assures that the parity of all majority comparisons is even and a corresponding preference profile consisting of strict preferences only exists. The above case distinction still applies in so far as only the first, second, and fourth case are still relevant, the third and fifth are ruled out by anti-symmetry.

Next, if $\succsim^{2}$ is anti-symmetric, $\succsim^{1}$ is not. Using the original weighting function guarantees odd parity for all majority comparisons and hence that we can find a preference profile consisting of strict preferences. Once more, the case distinction applies.

All in all, we have that every Fishburn-manipulation constitutes a Fishburn-majoritymanipulation with the required properties regarding the majority relations and given a Fishburn-majority-manipulation it is possible to construct a Fishburn-manipulation. This completes the proof.

Lemma 1 is useful since Fishburn-majority-participation can be encoded in propositional logic (with variables $f_{\succsim, X}$ representing $f(\succsim)=X$ ) much more concisely than Fishburnparticipation. To see this, let $\succsim$ and $\succsim^{\prime}$ be two majority relations. Then,

$$
\neg\left(f\left(\succsim^{\prime}\right) \succ_{i}^{F} f(\succsim)\right) \equiv \bigwedge_{Y \succ_{i}^{F} X}\left(\neg f_{\succsim, X} \vee \neg f_{\succsim^{\prime}, Y}\right)
$$

for all sets of alternatives $X, Y$ and all preference relations $\succsim_{i}$ satisfying conditions (1) to (3).

The size of the search space grows significantly with the introduction of weak majority relations (see Table 1). This larger search space can only be managed by an optimized encoding based on identifying isomorphic graphs (we do this via canonical representations). ${ }^{6}$

\subsection{Proof Extraction}

A very interesting feature of the approach by Brandt and Geist (2016) is the possibility to extract human-readable proofs from an unsatisfiability result by the SAT solver. This is done by computing a minimal unsatisfiable set (MUS), an inclusion-minimal set of clauses that is still unsatisfiable. ${ }^{7}$ This MUS can then, assisted by our encoder/decoder program, be read and transformed into a standard human-readable proof. Different proofs can be found by varying the MUS extractor or by encoding the problem for different subdomains, such as neighborhoods of a set of profiles or randomly sampled subdomains, respectively.

\section{Results and Discussion}

In general, participation and strategyproofness are not logically related. However, extending an observation by Brandt (2015), we show that strategyproofness implies participation under certain conditions.

6. Without this optimization, only domains of up to 4 alternatives can be solved within a reasonable time frame.

7. We used PicomUS, which is part of the PicoSAT distribution (Biere, 2008). 
Lemma 2. Consider an arbitrary preference extension. Every SCF that satisfies IIV and strategyproofness satisfies participation. Every majoritarian SCF that satisfies strategyproofness satisfies participation, even when preferences are strict.

Proof. We show both statements for Kelly's extension. The same argument works for any other preference extension, including Fishburn's.

For the first statement, let $f$ be an SCF that satisfies IIV and Kelly-strategyproofness. Assume for contradiction that $f$ is Kelly-manipulable by strategic abstention, i.e., there is a preference profile $R$ and an agent $i$ such that $f\left(R_{-i}\right) \succ_{i}^{K} f(R)$. Let $R^{\prime}$ be a preference profile such that $\succsim_{j}^{\prime}=\succsim_{j}$ for all $j \neq i$ and $\succsim_{i}^{\prime}$ the preference relation that expresses indifference between all alternatives, i.e., $x \sim_{i}^{\prime} y$ for all $x, y \in A$. Then, since $f$ satisfies IIV,

$$
f\left(R^{\prime}\right)=f\left(R_{-i}\right) \succ_{i}^{K} f(R),
$$

i.e., $i$ can manipulate in $R$ by reporting $\succsim_{i}^{\prime}$ instead of $\succsim_{i}$, which contradicts Kellystrategyproofness of $f$.

For the second statement, let $f$ be a majoritarian SCF that satisfies Kellystrategyproofness. Assume for contradiction that $f$ is Kelly-manipulable by strategic abstention, i.e., there is a preference profile $R$ and an agent $i$ such that $f\left(R_{-i}\right) \succ_{i}^{K} f(R)$. Let $2 R$ be a preference profile that consists of two copies of each preference relation in $R$, i.e., for every $\left(i, \succsim_{i}\right) \in R$ there are $\left(i_{1}, \succsim_{i_{1}}\right),\left(i_{2}, \succsim_{i_{2}}\right) \in 2 R$ such that $\succsim_{i}=\succsim_{i_{1}}=\succsim_{i_{2}}$. Note that $R$ and $2 R$ have the same majority relation. $2 R_{-i}$ is defined analogously.

We define a preference profile $2 R^{\prime}$ such that $2 \succsim_{j}^{\prime}=2 \succsim_{j}$ for all $j \neq i_{1}$ and $2 \succsim_{i_{1}}^{\prime}=\succsim_{i_{1}}^{-1}$, where $\succsim_{i_{1}}^{-1}$ denotes the inverse of $\succsim_{i_{1}}$, i.e., for all $x, y \in A, x \succsim_{i_{1}}^{-1} y$ if and only if $y \succsim_{i_{1}} x$. Intuitively, $2 R^{\prime}$ is thus the same as $2 R$ but for the preferences of agent $i_{1}$ which are reversed. In terms of the majority relation of $R^{\prime}$, we thus have that $i_{1}$ and $i_{2}$ cancel each other out, i.e., do not have an effect on the majority relation. Consequently, the majority relations of $2 R^{\prime}$ and $2 R_{-i}$ are identical. Then, since $f$ is majoritarian,

$$
f\left(2 R^{\prime}\right)=f\left(2 R_{-i}\right)=f\left(R_{-i}\right) \succ_{i}^{K} f(R)=f(2 R),
$$

i.e., $i_{1}$ can manipulate in $2 R$ by reporting $\succsim_{i_{1}}^{-1}$. This contradicts Kelly-strategyproofness of $f$. Note that the proof of the second statement only requires strict preferences.

As a consequence of Lemma 2, some positive results for Kelly-strategyproofness and Fishburn-strategyproofness carry over to participation. ${ }^{8}$ We will complement these results by impossibility theorems for Fishburn-participation and a positive result for Kellyparticipation, which specifically does not hold for Kelly-strategyproofness.

\subsection{Fishburn-Participation}

It turns out that Pareto-optimality is incompatible with Fishburn-participation for majoritarian SCFs. The corresponding Theorems 1 and 2 and their proofs were obtained using the computer-aided method laid out in Section 4. In order to simplify the original proofs, which were found by the computer, we first state a lemma that offers further insights into

8. See Table 2 in Section 6 for more details. 
the possible choices of majoritarian SCFs that satisfy Fishburn-participation and Paretooptimality.

To state Lemma 3 we introduce some additional notation: an alternative $x$ (McKelvey) covers an alternative $y$ if $x$ is at least as good as $y$ compared to every other alternative. Formally, $x$ covers $y$ if $x \succ y$ and, for all $z \in A$, both $y \succsim z$ implies $x \succsim z$, and $z \succsim x$ implies $z \succsim y$. The uncovered set of $\succsim$, denoted $U C(\succsim)$, is the set of all alternatives that are not covered by any other alternative. We inductively define $U C^{k}(\succsim)$ as the repeated application of $U C$, i.e., $U C^{1}(\succsim)=U C(\succsim)$ and $U C^{k}(\succsim)=U C\left(\left.\succsim\right|_{U C^{k-1}(\succsim)}\right)$ for $k>1$, where $\left.\succsim\right|_{U C^{k-1}(\succsim)}$ is the restriction of $\succsim$ to alternatives in $U C^{k-1}(\succsim)$. Then, the iterated uncovered set is defined by $U C^{\infty}(\succsim)=\bigcap_{k \geq 1} U C^{k}(\succsim)$. We provide examples for both $U C$ and $U C^{\infty}$ after the next lemma. By definition, $U C$ and $U C^{\infty}$ are majoritarian SCFs.

Brandt, Geist, and Harrenstein (2016) proved that every majoritarian and Paretooptimal SCF selects a subset of the (McKelvey) uncovered set. We show that an SCF that additionally satisfies Fishburn-participation only chooses alternatives from the iterated uncovered set. Furthermore, this choice only depends on the majority relation between alternatives within the iterated uncovered set whenever the majority relation is strict.

Lemma 3. Let $f$ be a majoritarian and Pareto-optimal SCF that satisfies Fishburnparticipation. Let $R, R^{\prime}$ be preference profiles such that $\left.\succsim_{R}\right|_{U C^{\infty}\left(\succsim_{R}\right)}=\left.\succsim_{R^{\prime}}\right|_{U C^{\infty}\left(\succsim_{R^{\prime}}\right)}$. Then $f(R) \subseteq U C^{\infty}\left(\succsim_{R}\right)$ and if $\succsim_{R}$ and $\succsim_{R^{\prime}}$ are additionally antisymmetric, we have that $f(R)=f\left(R^{\prime}\right) \cdot{ }^{9}$

Proof. We begin the proof by showing that $f(R) \subseteq U C^{\infty}\left(\succsim_{R}\right)$. Let $f$ be a majoritarian and Pareto-optimal SCF that satisfies Fishburn-participation, $N \in \mathcal{F}(\mathbb{N})$ a set of agents, $R \in \mathcal{R}^{N}$ a preference profile and $\succsim_{R}$ the majority relation of $R$. We prove inductively that $f(R) \subseteq U C^{k}\left(\succsim_{R}\right)$ for all $k \in \mathbb{N}$.

First, let $k=1$. Brandt et al. (2016) have shown that if an alternative $x$ is not in the McKelvey uncovered set $U C\left(\succsim_{R}\right)$, it is potentially Pareto-dominated, i.e., there is a preference profile $R^{\prime}$ such that $\succsim_{R}=\succsim_{R^{\prime}}$ and $x$ is Pareto-dominated in $R^{\prime}$. Hence, $x \notin f\left(R^{\prime}\right)$ and as well $x \notin f(R)$, since $f$ is majoritarian and Pareto-optimal.

Now let $k \geq 2$. By induction, $f(R) \subseteq U C^{k-1}\left(\succsim_{R}\right)$. If $U C^{k-1}\left(\succsim_{R}\right)=U C^{k}\left(\succsim_{R}\right)$, there is nothing left to show. Hence, we consider the remaining case, i.e., $U C^{k}\left(\succsim_{R}\right) \subsetneq U C^{k-1}\left(\succsim_{R}\right)$. By Proposition 1, we can find a preference profile $R^{\prime} \in \mathcal{R}^{N^{\prime}}$ such that $\succsim_{R^{\prime}}=\succsim_{R}$ and

$$
\left|w_{R^{\prime}}(x, y)\right|= \begin{cases}\leq 1 & \text { for all } x \in U C^{k}\left(\succsim_{R}\right), y \in A \backslash U C^{k-1}\left(\succsim_{R}\right), \text { and } \\ \geq 3 & \text { otherwise. }\end{cases}
$$

This intuitively means that one or two joining agents can only affect majority comparisons in between alternatives $x$ and $y$ where $x \in U C^{k}\left(\succsim_{R}\right)$ and $y \in A \backslash U C^{k-1}\left(\succsim_{R}\right)$. Note, in particular, that if two agents $j, j^{\prime}$ with $x \succsim_{j} y, x \succsim_{j^{\prime}} y$ for all such $x, y$ join the electorate, we have that every alternative in $U C^{k}\left(\succsim_{R}\right)$ is majority-preferred to every alternative in $A \backslash U C^{k-1}\left(\succsim_{R}\right)$.

9. Lemma 3 can be strengthened in various respects and also holds for all other preference extensions satisfying some mild conditions as well as probabilisitic social choice functions. 
Let $\succsim_{i}$ be a preference relation such that $x \succsim_{i} y$ for all alternatives $x \in U C^{k}\left(\succsim_{R^{\prime}}\right)$ and $y \in A \backslash U C^{k-1}\left(\succsim_{R^{\prime}}\right)$. Clearly, $U C^{k-1}\left(\succsim_{R_{+i}^{\prime}}\right) \subseteq U C^{k-1}\left(\succsim_{R^{\prime}}\right)$ as nothing covered before can become uncovered.

If $f\left(R^{\prime}\right) \neq f\left(R_{+i}^{\prime}\right)$, we can find some $\succsim_{j}$ such that $x \succsim_{j} y$ for all $x \in U C^{k}\left(\succsim_{R^{\prime}}\right)$ and $y \in A \backslash U C^{k-1}\left(\succsim_{R^{\prime}}\right)$ and $f\left(R^{\prime}\right) \succ_{j}^{F} f\left(R_{+j}^{\prime}\right)$, which contradicts Fishburn-participation. Thus, $f\left(R^{\prime}\right)=f\left(R_{+i}^{\prime}\right)$. With the same reasoning we can find $\succsim_{j^{\prime}}$ such that $x \succsim_{j^{\prime}} y$ for all alternatives $x \in U C^{k}\left(\succsim_{R^{\prime}}\right)$ and $y \in A \backslash U C^{k-1}\left(\succsim_{R^{\prime}}\right)$ and $f\left(R_{+j, j^{\prime}}^{\prime}\right)=f\left(R_{+j}^{\prime}\right)=f\left(R^{\prime}\right)$.

Using the result by Brandt et al. (2016) again, Pareto-optimality of $f$ directly implies that $f\left(R_{+j, j^{\prime}}^{\prime}\right) \subseteq U C\left(\succsim_{R_{+j, j^{\prime}}}\right)$. By definition of $R^{\prime}, \succsim_{j}$ and $\succsim_{j^{\prime}}$, we have that $U C\left(\succsim_{R_{+j, j^{\prime}}^{\prime}}\right)=U C^{k}\left(\succsim_{R^{\prime}}\right)$. Hence,

$$
f(R)=f\left(R^{\prime}\right)=f\left(R_{+j, j^{\prime}}\right) \subseteq U C\left(\succsim_{R_{+j, j^{\prime}}^{\prime}}\right)=U C^{k}\left(\succsim_{R^{\prime}}\right)=U C^{k}\left(\succsim_{R}\right),
$$

which completes the first part of the proof.

Next, let $f$ be as above and $R$ and $R^{\prime}$ two preference profiles on some agenda $A$ such that $\left.\succsim_{R}\right|_{U C^{\infty}\left(\succsim_{R}\right)}=\left.\succsim_{R^{\prime}}\right|_{U C^{\infty}\left(\succsim_{R^{\prime}}\right)}$. Suppose for contradiction that $f(R) \neq f\left(R^{\prime}\right)$. Since $f$ is majoritarian define without loss of generality for $\succsim_{R}$ majority margins $|w(x, y)|$ suitably

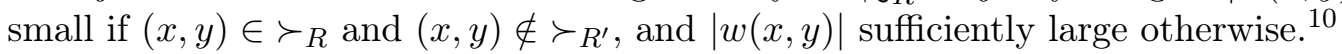

We successively include $k$ agents $i_{1}, \ldots, i_{k}$ into the corresponding electorate, all with individual preferences such that all alternatives in $U C^{\infty}\left(\succsim_{R^{\prime}}\right)$ are adjacent in $\succsim_{i_{j}}$. All other alternatives have to be ranked such that $\succsim_{R_{\left\{+i_{1}, \ldots, i_{k}\right\}}}=\succsim_{R^{\prime}}$, yielding that $f\left(R_{\left\{+i_{1}, \ldots, i_{k}\right\}}\right) \neq f(R)$. This is possible because we assumed the weights of all edges that have to be changed to be suitably small. Since the ordering of alternatives within $U C^{\infty}\left(\succsim_{R^{\prime}}\right)$ does not affect the majority relation due to sufficiently large edge weights, we are able to arrange them such that $x \succ_{i_{j}} y \succ_{i_{j}} z$ for all

$$
\begin{aligned}
& x \in f\left(R_{\left\{+i_{1}, \ldots, i_{j-1}\right\}}\right) \backslash f\left(R_{\left\{+i_{1}, \ldots, i_{j}\right\}}\right), \\
& y \in f\left(R_{\left\{+i_{1}, \ldots, i_{j-1}\right\}}\right) \cap f\left(R_{\left\{+i_{1}, \ldots, i_{j}\right\}}\right), \text { and } \\
& z \in U C^{\infty}\left(\succsim_{R}\right) \backslash f\left(R_{\left\{+i_{1}, \ldots, i_{j-1}\right\}}\right),
\end{aligned}
$$

$1 \leq j \leq k$. Intuitively, this means that everything that was in the choice set before $i_{j}$ joined the electorate but is not contained anymore is preferred to everything that still is in the choice set is preferred to everything that is possibly chosen now but was not chosen before. Thus, every agent $i_{j}$ prefers what was chosen without him to what is chosen including him.

Hence, for at least one agent $i_{j}, 1 \leq j \leq k$, it has to hold that $f\left(R_{+i_{1}, \ldots, i_{j-1}}\right) \neq f\left(R_{+i_{1}, \ldots, i_{j}}\right)$ and by definition of this agent's preferences $f\left(R_{+i_{1}, \ldots, i_{j-1}}\right) \succ_{i_{j}}^{F} f\left(R_{+i_{1}, \ldots, i_{j}}\right)$. This contradicts Fishburn-participation and completes the proof.

10. Since $\succsim_{R}$ and $\succsim_{R^{\prime}}$ are antisymmetric we obviously have $w(x, y) \neq 0$ for all $x, y \in A$. 
The following brief example illustrates an application of Lemma 3 . Let $R$ be a preference profile on $A=\{a, b, c, d, e\}$ such that $\succsim_{R}$ is as depicted below. We have that $U C\left(\succsim_{R}\right)=\{a, b, d, e\}$ and $U C^{2}\left(\succsim_{R}\right)=U C^{\infty}\left(\succsim_{R}\right)=$ $\{a, b, d\}$. For all majoritarian and Pareto-optimal SCFs $f$ satisfying Fishburn-participation, Lemma 3 gives that $f(R) \subseteq\{a, b, d\}$. Also, the choice set has to be identical to the one of any other profile with identically structured iterated uncovered set, e.g., a profile $R^{\prime}$ where the iterated uncovered set is a three-cycle on $\{a, b, d\}$. Thus, we even have $f(R)=\{a, b, d\}$ due to neutrality. Similar arguments are used repeatedly in the proofs of Theorem 1 and Theorem 2 .

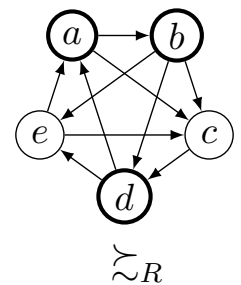

Now, let us turn to our impossibility theorems. The computer found these impossibilities even without using Lemma 3. However, the formalization of the lemma allowed the SAT solver to find smaller proofs and makes the human-readable proofs more intuitive.

Theorem 1. There is no majoritarian and Pareto-optimal SCF that satisfies Fishburnparticipation if $|A| \geq 4$ and $|N| \geq 6$.

Proof. Let $f$ be a majoritarian and Pareto-optimal SCF satisfying Fishburn-participation. Note that majoritarianness implies anonymity and neutrality. We first prove the statement for $A=\{a, b, c, d\}$ and reason about the outcome of $f$ for some specific majority relations. Throughout this proof, we are going to make extensive use of Lemma 1, which allows us to apply Fishburn-majority-participation instead of regular Fishburn-participation. Intuitively, the proof strategy is to alter the majority relations $\succsim, \succsim^{\prime}$, and $\succsim^{\prime \prime}$ as depicted below by letting varying agents join some underlying electorate, which will exclude certain choices of $f$ (by an application of Fishburn-majority-participation), until we reach a contradiction. For each step, i.e., each time an additional agent alters one of the majority relations, we provide a suitable electorate inducing $\succsim, \succsim^{\prime}$, and $\succsim^{\prime \prime}$. Consider for instance $\succsim$, which can be altered to $\succsim_{+1}$ by an agent with preferences $\succsim_{1}:\{a, b, c\}, d$. For this to be possible, we need an underlying electorate where $d$ has to be majority-preferred to $b$ by a margin of exactly one, while it has to be preferred to $a$ by a margin of at least two. These constraints make the construction of suitable electorates more demanding with respect to the number of agents needed compared to the case where only the simple majority relation is of relevance. In the figures depicting the strict part of the majority relations we highlight alternatives that have to be chosen by $f$ with a thick border.

First consider $\succsim$ as depicted on the right. In the following, arguments using the additional agent's preferences as well as which effects him joining has on the set of possibly chosen alternatives are given on the left, while suitable electorates inducing $\succsim$ together with the changed majority graph are given on the right. Whenever an electorate works for multiple cases they are grouped in a single paragraph with the preference profile

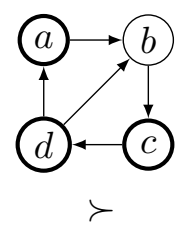
next to it. 
Adding an agent with preferences $\succsim_{1}:\{a, b, c\}, d$ yields $\succsim_{+1}$ where, due to symmetry, $f\left(\succsim_{+1}\right)=\{a, b, c, d\}$. As $f$ satisfies Fishburn-participation, nothing that is strictly preferred to

\begin{tabular}{cccc}
1 & 2 & 3 & 4 \\
\hline$a$ & $b$ & $c$ & $d$ \\
$b, c, d$ & $c$ & $d$ & $a$ \\
& $d$ & $a$ & $b$ \\
& $a$ & $b$ & $c$
\end{tabular}
$\{a, b, c, d\}$ according to $\succsim_{1}^{F}$ can be chosen in $\succsim$. Thus, $d \in f(\succsim)$. Adding another agent with $\succsim_{1^{\prime}}: b,\{a, c, d\}$ also leads to $\succsim_{+1}$. Hence, $f(\succsim) \neq\{b, d\},\{a, b, d\},\{b, c, d\}$.

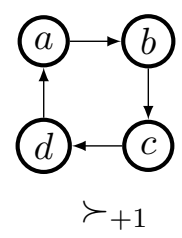

If $\succsim$ is altered to $\succsim+2$ by adding an agent with $\succsim_{2}:\{b, d\}, c, a$, we get $f\left(\succsim_{+2}\right)=\{b, c, d\}$, by the fact that $a$ is covered by $d$ together with neutrality. Therefore, $f(\succsim) \neq\{d\},\{c, d\}$. Note that here $|N|=5$ is the minimal number of other agents needed to construct a suitable preference profile.

Adding an agent with $\succsim_{3}:\{a, b, d\}, c$ leads to $\succsim_{+3}$, for which we have $f\left(\succsim_{+3}\right)=\{a, c, d\}$ by the fact that $b$ is covered by $a$ together with neutrality. We correspondingly deduce $f(\succsim) \neq\{a, d\},\{a, b, c, d\}$. Hence, we conclude that $f(\succsim)=\{a, c, d\}$.

\begin{tabular}{ccccc}
1 & 2 & 3 & 4 & 5 \\
\hline$a$ & $a, c$ & $b$ & $c$ & $d$ \\
$b$ & $d$ & $c$ & $d$ & $a$ \\
$c$ & $b$ & $d$ & $a$ & $b$ \\
$d$ & & $a$ & $b$ & $c$
\end{tabular}
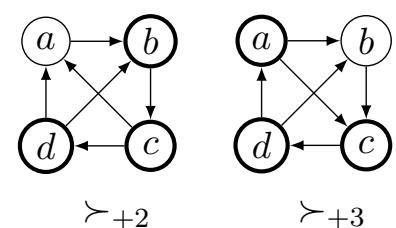

Now consider $\succsim^{\prime}$ as shown on the right. Neutrality implies that $f\left(\succsim^{\prime}\right)$ contains either none or both of $a$ and $b$.

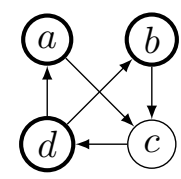

$\succ^{\prime}$

Adding an agent with preferences $\succsim_{1}^{\prime}: c, a, b, d$ changes $\succsim^{\prime}$ to $\succsim_{+1}^{\prime}$ where $\{a, b, c, d\}$ is selected. Thus, $f\left(\succsim^{\prime}\right) \neq\{c\},\{a, b, c\}$.

Additionally, an agent with $\succsim_{2}^{\prime}:\{c, d\}, a, b$, alters $\succsim^{\prime}$ to $\succsim_{+2}^{\prime}$. Hence, $f\left(\succsim^{\prime}\right) \neq\{d\},\{c, d\}$.

Adding an agent with $\succsim_{3}^{\prime}:\{b, c\}, a, d$ changes $\succsim^{\prime}$ to $\succsim_{+3}^{\prime}$. $\succsim_{+3}^{\prime}$ is isomorphic to $\succsim$ which implies $f\left(\succsim_{+3}^{\prime}\right)=\{a, b, d\}$. Thus, $f\left(\succsim^{\prime}\right) \neq\{a, b\},\{a, b, c, d\}$. Putting everything together, we deduce that $f\left(\succsim^{\prime}\right)=\{a, b, d\}$.

\begin{tabular}{cccc}
1 & 2,3 & 4 & 5 \\
\hline$a$ & $b$ & $d$ & $d$ \\
$c$ & $c$ & $a$ & $a, b$ \\
$d$ & $d$ & $b$ & $c$ \\
$b$ & $a$ & $c$ &
\end{tabular}

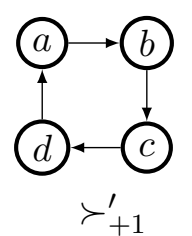

\begin{tabular}{ccccc}
1 & 2 & 3 & 4 & 5 \\
\hline$a$ & $a, b$ & $b$ & $d$ & $d$ \\
$c$ & $c$ & $c$ & $a$ & $b$ \\
$d$ & $d$ & $d$ & $b$ & $a$ \\
$b$ & & $a$ & $c$ & $c$
\end{tabular}

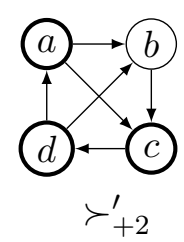

\begin{tabular}{cccc}
1 & 2 & 3 & 4,5 \\
\hline$a$ & $a$ & $c$ & $d$ \\
$b$ & $c$ & $d$ & $b$ \\
$c$ & $d$ & $a, b$ & $a$ \\
$d$ & $b$ & & $c$
\end{tabular}

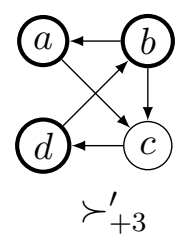


Finally consider $\succsim^{\prime \prime}$ as depicted on the right. Neutrality implies that $f\left(\succsim^{\prime \prime}\right)$ contains either none or both of $b$ and $c$.
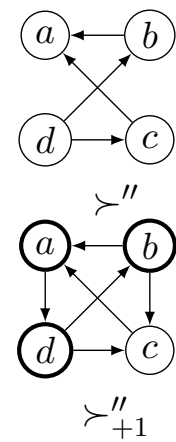

Adding an agent with $\succsim_{1}^{\prime \prime}: a,\{b, d\}, c$ changes $\succsim^{\prime \prime}$ to $\succsim_{+1}^{\prime \prime}$. Analogously to $\succsim_{+2}, f\left(\succsim_{+1}^{\prime \prime}\right)=\{a, b, d\}$. Therefore, $f\left(\succsim^{\prime \prime}\right) \neq\{a, d\}$.

Adding an agent with preferences $\succsim_{2}^{\prime \prime}: c,\{b, d\}, a$ alters $\succsim^{\prime \prime}$ to $\succsim_{+2}^{\prime \prime}$. Alternative $d$ is a Condorcet winner in $\succsim_{+2}^{\prime \prime}$, which implies that $f\left(\succsim_{+2}^{\prime \prime}\right)=\{d\}$ because $d$ covers $a, b$, and $c$. Hence, $f\left(\succsim^{\prime \prime}\right) \neq\{b, c\},\{b, c, d\}$.

Both an agent with $\succsim_{3}^{\prime \prime}: a, d,\{b, c\}$ as well as with $\succsim_{3^{\prime}}^{\prime \prime}:\{a, b, c\}, d$, can alter $\succsim^{\prime \prime}$ to $\succsim_{+3}^{\prime \prime}$ which is isomorphic to $\succsim^{\prime}$. Thus, $f\left(\succsim_{+3}^{\prime \prime}\right)=\{b, c, d\}$. This implies that $f\left(\succsim^{\prime \prime}\right) \neq\{d\},\{a, b, c, d\}$, otherwise an agent with $\succsim_{3}^{\prime \prime}$ or $\succsim_{3^{\prime}}^{\prime \prime}$, respectively, could manipulate.

\begin{tabular}{llll}
1 & 2 & 3 & 4 \\
\hline$b$ & $c$ & $d$ & $d$ \\
$a$ & $a$ & $b$ & $c$ \\
$d$ & $d$ & $c$ & $b$ \\
$c$ & $b$ & $a$ & $a$
\end{tabular}
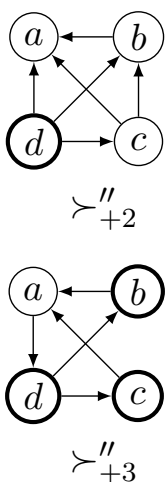

An agent with $\succsim_{4}^{\prime \prime}: a, b, c, d$ changes $\succsim^{\prime \prime}$ to $\succsim_{+4}^{\prime \prime}$. Neutrality implies that $f\left(\succsim_{+4}^{\prime \prime}\right)=\{a, b, c, d\}$. This gives that $f\left(\succsim^{\prime \prime}\right) \neq\{a\},\{a, b, c\}$.

Consequently, if Fishburn-participation is to be respected, $f$ cannot choose anything from $\succsim^{\prime \prime}$, a contradiction.

\begin{tabular}{cccc}
1 & 2 & 3 & 4 \\
\hline$a, b$ & $c$ & $d$ & $d$ \\
$c, d$ & $a$ & $b$ & $d$ \\
& $d$ & $c$ & $b$ \\
& $b$ & $a$ & $a$
\end{tabular}

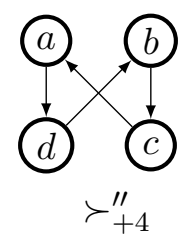

Now let $|A| \geq 5$. It follows from Lemma 3 that the choice of $f$ does not depend on covered alternatives. Hence the statement follows by extending the majority relations depicted above to $A$ such that all alternatives but $a, b, c, d$ are covered (for instance, by adding Condorcet losers only).

The proof of Theorem 1 requires ties in the agents' preferences. Theorem 2 shows that this impossibility still holds for strict preferences when there are at least five alternatives and seven agents. The steps in the human-readable proof of Theorem 2 are similar to those in the proof of Theorem 1. We therefore defer the lengthy proof to the appendix.

Theorem 2. There is no majoritarian and Pareto-optimal SCF that satisfies Fishburnparticipation if $|A| \geq 5$ and $|N| \geq 7$, even when preferences are strict.

Theorems 1 and 2 are both tight in the sense that, whenever there are less than four or five alternatives, respectively, there exists an SCF that satisfies the desired properties.

An interesting question is whether these impossibilities also extend to other preference extensions. Given the computer-aided approach, this can be easily checked by simply replacing the preference extension in the SAT encoder. For instance, it turns out that the impossibility of Theorem 1 still holds for a coarsening of Fishburn's extension which only 
allows the comparison of sets that are contained in each other. Kelly's extension on the other hand does not lead to an impossibility for $|A| \leq 5$, which will be confirmed by a general positive result in the next section.

\subsection{Kelly-Participation}

Theorems 1 and 2 are sweeping impossibilities within the domain of majoritarian SCFs. For Kelly's extension, we obtain a positive result that covers attractive majoritarian and non-majoritarian SCFs. Brandt (2015) has shown that set-monotonicity implies Kelly-strategyproofness for strict preferences and that no Condorcet extension is Kellystrategyproof when preferences are weak. We prove that set-monotonicity (and the very mild IIV axiom) imply Kelly-participation even for weak preferences. We have thus found natural examples of SCFs that violate Kelly-strategyproofness but satisfy Kellyparticipation.

Theorem 3. Let $f$ be an SCF that satisfies IIV and set-monotonicity. Then $f$ satisfies Kelly-participation.

Proof. Let $f$ be an SCF that satisfies IIV and set-monotonicity. Assume for contradiction that $f$ does not satisfy Kelly-participation. Hence there exist a preference profile $R$ and an agent $i$ such that $f\left(R_{-i}\right) \succ_{i}^{K} f(R)$. Let $X=f(R), Y=f\left(R_{-i}\right)$, and $Z=A \backslash(X \cup Y)$. By definition of $Y \succ_{i}^{K} X$ we have that $x \sim_{i} y$ for all $x, y \in X \cap Y$.

We define a new preference relation $\succsim_{i^{\prime}}$ in which all alternatives in $Y$ are tied for the first place, followed by all alternatives in $X \backslash Y$ (which may be empty) as they are ordered in $\succsim_{i}$, and all remaining alternatives in one indifference class at the bottom of the ranking. Formally,

$$
\succsim_{i^{\prime}}=\left.(Y \times A) \cup \succsim_{i}\right|_{X} \cup(A \times Z) .
$$

Let $i^{\prime \prime}$ be an agent who is indifferent between all alternatives, i.e., $x \sim_{i^{\prime \prime}} y$ for all $x, y \in A$. Since $f$ satisfies IIV we have that $f\left(R_{-i+i^{\prime \prime}}\right)=f\left(R_{-i}\right)$.

By definition, $R_{-i+i^{\prime}}$ is an $f$-improvement over both $R$ and $R_{-i+i^{\prime \prime}}$. Hence, setmonotonicity implies that $f\left(R_{-i+i^{\prime}}\right)=f(R)$ and $f\left(R_{-i+i^{\prime}}\right)=f\left(R_{-i+i^{\prime \prime}}\right)$. In summary, we obtain

$$
f\left(R_{-i+i^{\prime}}\right)=f\left(R_{-i+i^{\prime \prime}}\right)=f\left(R_{-i}\right) \succ_{i}^{K} f(R)=f\left(R_{-i+i^{\prime}}\right),
$$

which is a contradiction.

Inspecting the proof of Theorem 3 shows that it extends to group-participation, i.e., no group of agents can obtain a unanimously more preferred outcome by abstaining. This can be seen by applying the same construction to all agents in the manipulating group simultaneously.

Two rather undiscriminating SCFs that satisfy both IIV and set-monotonicity are the Pareto rule and the omninomination rule (which returns all alternatives that are ranked first by at least one agent). Majoritarian SCFs satisfy IIV by definition and there are several appealing majoritarian SCFs that satisfy set-monotonicity such as the top cycle, (also known as weak closure maximality, GETCHA, or the Smith set) (Good, 1971; Smith, 1973; Bordes, 1976; Sen, 1977; Schwartz, 1986), the minimal covering set (Dutta, 1990), the 
bipartisan set (see Laffond, Laslier, \& Le Breton, 1993; Brandt, 2015; Brandt et al., 2016) and variations thereof (see Laslier, 1997; Dutta \& Laslier, 1999; Laslier, 2000; Brandt, 2011). These majoritarian SCFs are sometimes criticized for not being discriminating enough. ${ }^{11}$

The computer-aided approach described in this paper can be used to find more discriminating SCFs that still satisfy Kelly-participation. We thus found a refinement of the bipartisan set that, for $|A|=5$, selects only 1.43 alternatives on average, and satisfies Kelly-participation. For comparison, the bipartisan set (the smallest previously known majoritarian SCF satisfying Kelly-participation) yields 2.68 alternatives on average.

\section{Conclusion}

Previous results have indicated a conflict between strategic non-manipulability and Condorcet-consistency (Moulin, 1988; Pérez, 2001; Jimeno et al., 2009; Brandt, 2015). In particular, Moulin (1988) has shown that no resolute Condorcet extension satisfies participation and Brandt (2015) has shown that no irresolute Condorcet extension satisfies Kelly-strategyproofness for weak preferences. Theorem 3 addresses an intermediate question and finds that - perhaps surprisingly - there are attractive Condorcet extensions that satisfy Kelly-participation, even when preferences are weak. On the other hand, we have presented elaborate computer-generated impossibilities (Theorems 1 and 2), which show that these encouraging results break down once preferences are extended by the more refined Fishburn extension. These findings improve our understanding of which assumptions allow for aggregation functions that are immune to strategic abstention. For example, one may assume that an agent prefers one choice set to another if the former yields more expected utility than the latter for all utility representations consistent with the ordinal preferences. In this model, Theorems 1 and 2 imply impossibilities when ties are broken by uniform randomization and Theorem 3 represents a positive result for the case when tie-breaking probabilities are unknown. An overview of the main results of this paper and how they relate to results for strategyproofness is given in Table 2 .

For Fishburn's extension, it would be interesting to closer examine how the picture changes when giving up majoritarianness, for example by considering the more general class of pairwise SCFs, i.e., SCFs whose output only depends on the majority margins. In particular, the question is whether there is a pairwise SCF satisfying Condorcet-consistency, Pareto-optimality, and Fishburn-participation. For any combination of three of these four properties, we can give an affirmative answer.

- The uncovered set is majoritarian (and hence pairwise), Condorcet-consistent, and Pareto-optimal (Fishburn, 1977). It does, however, violate Fishburn-participation (Theorem 1 and Theorem 2). The same is true for other well-known pairwise rules such as the essential set, Nanson's rule, Kemeny's rule, Schulze's rule, and ranked pairs.

- The SCF that selects the Condorcet winner whenever one exists and all alternatives otherwise is majoritarian (and hence pairwise), Condorcet-consistent, and satisfies

11. Indeed, Scott and Fey (2012) show that the minimal covering set selects all alternatives in almost all large tournaments. The bipartisan set selects half of the alternatives on average (Fisher \& Reeves, 1995). The question of discriminability has been addressed in detail by Brandt and Seedig (2016). 


\begin{tabular}{|c|c|c|c|}
\hline & & Strict preferences & Weak preferences \\
\hline Kelly-Participation & $\oplus$ & \multicolumn{2}{|c|}{ All set-monotonic SCFs that satisfy IIV (Theorem 3) } \\
\hline Kelly-Strategyproofness & $\oplus$ & All set-monotonic $\mathrm{SCFs}^{a}$ & $\ominus \begin{array}{l}\text { No Condorcet extension } \\
\text { no pairwise \& } \\
\text { Pareto-optimal SCF } \\
(|A|,|N| \geq 3)^{d}\end{array}$ \\
\hline \multirow{2}{*}{ Fishburn-Participation } & $\ominus$ & $\begin{array}{l}\text { No majoritarian \& } \\
\text { Pareto-optimal SCF } \\
(|A| \geq 5,|N| \geq 7) \\
\text { (Theorem 2) }\end{array}$ & $\ominus \begin{array}{l}\text { No majoritarian \& } \\
\text { Pareto-optimal SCF } \\
(|A| \geq 4,|N| \geq 6) \\
(\text { Theorem 1) }\end{array}$ \\
\hline & $\oplus$ & 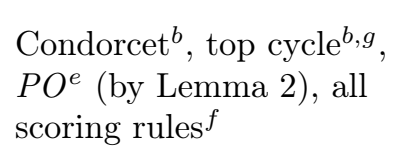 & \multirow{3}{*}{$\ominus \begin{array}{l}\text { No anonymous \& } \\
\text { Pareto-optimal SCF } \\
(|A|,|N| \geq 3)^{d}\end{array}$} \\
\hline \multirow{2}{*}{ Fishburn-Strategyproofness } & $\ominus$ & $\begin{array}{l}\text { No majoritarian \& } \\
\text { Pareto-optimal SCF } \\
(|A| \geq 5,|N| \geq 7)^{c}\end{array}$ & \\
\hline & $\oplus$ & $\begin{array}{l}\text { Condorcet }^{b}, \text { top cycle } \\
P O^{b, g}\end{array}$ & \\
\hline
\end{tabular}

Table 2: Overview of results for participation and strategyproofness with respect to Kelly's and Fishburn's extension and strict/weak preferences. The symbol $\oplus$ marks positive results while $\ominus$ marks impossibility results. The Condorcet SCF returns the Condorcet winner whenever one exists and all alternatives otherwise. The Top Cycle consists of all maximal elements of the transitive closure of the majority relation. ${ }^{a}$ : Brandt (2015), ${ }^{b}$ : Brandt and Brill (2011), ${ }^{c}$ : Brandt and Geist (2016), ${ }^{d}$ : Brandt et al. (2018), ${ }^{e}$ : Feldman (1979), ${ }^{f}$ : Moulin (1988), ${ }^{g}$ : Sanver and Zwicker (2012)

Fishburn-participation (Gärdenfors, 1976; Brandt \& Brill, 2011). In the absence of a Condorcet winner, it may select Pareto-dominated alternatives, though.

- Borda's rule is pairwise, Pareto-optimal, and satisfies Fishburn-participation (Moulin, 1988). On the other hand, it is well-known that it may fail to choose the Condorcet winner.

- The SCF that select selects the Condorcet winner whenever one exists and all Paretooptimal alternatives otherwise satisfies Condorcet-consistency, Pareto-optimality and Fishburn-participation (Brandt \& Brill, 2011). However, it is not pairwise.

If we require all four properties, the situation is, to the best of our knowledge, unclear. In general, it is interesting to observe that, for weak preferences, Pareto-optimality and 
Condorcet-consistency are compatible with Fishburn-participation while each of these properties is incompatible with Fishburn-strategyproofness.

\section{Acknowledgments}

The authors thank Christian Stricker and the anonymous reviewers for helpful comments. This material is based upon work supported by Deutsche Forschungsgemeinschaft under grants BR 2312/7-2, BR 2312/10-1, BR 2312/11-1, and BR 5969/1-1. Preliminary results of this paper were presented at the Dagstuhl Seminar on Computational Social Choice (Dagstuhl, Germany, June 2015) and the 24th International Joint Conference on Artificial Intelligence (Buenos Aires, Argentina, July 2015).

\section{Appendix A. Proof of Theorem 2}

Theorem 2. There is no majoritarian and Pareto-optimal SCF that satisfies Fishburnparticipation if $|A| \geq 5$ and $|N| \geq 7$, even when preferences are strict.

Proof. Let $f$ be a majoritarian and Pareto-optimal SCF satisfying Fishburn-participation and note, just as before, that majoritarianness implies anonymity and neutrality. We first prove the statement for $A=\{a, b, c, d, e\}$ and reason about the outcome of $f$ for some specific majority relations. Throughout this proof, we again make extensive use of Lemma 1, which allows us to apply Fishburn-majority-participation instead of regular Fishburn-participation. Intuitively, the basic proof structure is similar, but more intricate, than that of Theorem 1.

Briefly summarized, we focus on $\succsim^{1}$ as given on the rightessentially a 3 -cycle within a 3 -cycle. By neutrality we know that $f$ has to select either all of $c, d, e$ or none of them, i.e., there are only seven possible subsets of $A$ that $f$ can choose in $\succsim^{1}$ : $\{a\},\{b\},\{c, d, e\},\{a, b\},\{a, c, d, e\},\{b, c, d, e\}$ or $A$.

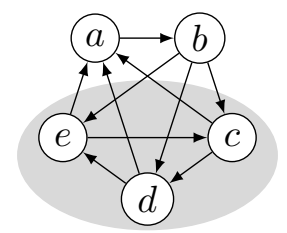

Using several auxiliary majority relations, we successively show that if $f$ selects $\{b\}$ or $\{a, b\}$, nothing can be chosen in $\succsim^{2}$, if $f$ selects $\{a, c, d, e\}$ or $A$, nothing can be chosen in $\succsim^{3}$, if $f$ selects $\{a\}$, nothing can be chosen in $\succsim^{4}$ and finally if $f$ chooses $\{c, d, e\}$ or $\{b, c, d, e\}$, nothing can be chosen in $\succsim^{5}$. Thus, whatever $f$ selects in $\succsim^{1}$, one of the other four majority relations is left without a possible choice set, a contradiction.

To begin with, consider the majority relation $\succsim^{2}$ as depicted on the right together with a preference profile inducing $\succsim^{2}$. Similar to before, we now let different agents join this electorate and examine which conclusions they allow with respect to $f\left(\succsim^{2}\right)$.

\begin{tabular}{ccccc}
1 & 2 & 3 & 4,5 & 6 \\
\hline$b$ & $b$ & $c$ & $d$ & $e$ \\
$c$ & $e$ & $a$ & $a$ & $a$ \\
$d$ & $c$ & $b$ & $b$ & $b$ \\
$e$ & $d$ & $d$ & $e$ & $c$ \\
$a$ & $a$ & $e$ & $a$ & $d$
\end{tabular}

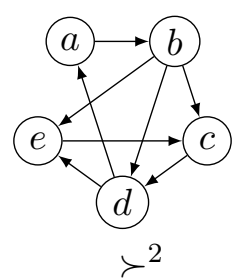


First, assume an agent with preferences $\succsim_{1}^{2}: e, b, a, d, c$ joins the electorate and consequently alters $\succsim^{2}$ to $\succsim_{+1}^{2}$. Here, we have that $f\left(\succsim_{+1}^{2}\right)=\{a, b, d\}$ according to Lemma 3. As nothing preferred to $\{a, b, d\}$ can be selected by $f$ in $\succsim^{2}$, we obtain $f\left(\succsim^{2}\right) \neq\{b\},\{a, b\}$.

If, on the other hand, either an agent with preferences $\succsim_{2}^{2}: d, c, e, b, a$ or an agent with preferences $\succsim_{2^{\prime}}^{2}: d, c, e, a, b$ joins the electorate, $\succsim^{2}$ is changed to $\succsim_{+2}^{2}$, which is equivalent to $\succsim^{1}$. Suppose for the following arguments, that $f$ chooses $\{b\}$ or $\{a, b\}$ in $\succsim^{1}$. With respect to $\succsim_{2}^{2}$ we thus obtain that $f\left(\succsim^{2}\right) \neq\{c\},\{d\},\{e\},\{b, c\},\{b, d\},\{b, e\},\{c, d\}$, $\{c, e\},\{d, e\},\{b, c, d\},\{b, c, e\},\{b, d, e\},\{c, d, e\},\{b, c, d, e\}$.

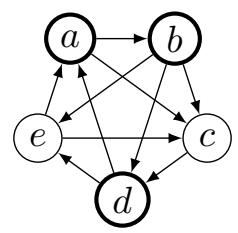

$\succ_{+1}^{2}$

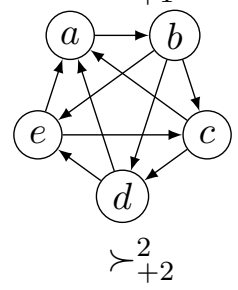

Similarly, when considering $\succsim_{2^{\prime}}^{2}$, we can deduce that $f\left(\succsim^{2}\right) \neq\{a\},\{a, c\},\{a, d\},\{a, e\}$, $\{a, b, c\},\{a, b, d\},\{a, b, e\},\{a, c, d\},\{a, c, e\},\{a, d, e\},\{a, b, c, d\},\{a, b, c, e\},\{a, b, d, e\}$, $\{a, c, d, e\}, A$. Put differently, we thus have that if $f\left(\succsim_{+2}^{2}\right)=f\left(\succsim^{1}\right)$ is either $\{b\}$ or $\{a, b\}$, then we obtain $f\left(\succsim^{2}\right)=\emptyset$. Consequently, $f\left(\succsim^{1}\right) \neq\{b\},\{a, b\}$.

Next, consider the majority relation $\succsim^{3}$ as given on the right together with a preference profile inducing $\succsim^{3}$. We proceed with our standard procedure of letting agents join this electorate.

\begin{tabular}{llllll}
1 & 2 & 3 & 4 & 5 & 6 \\
\hline$a$ & $b$ & $b$ & $c$ & $d$ & $d$ \\
$e$ & $c$ & $e$ & $a$ & $e$ & $e$ \\
$b$ & $d$ & $c$ & $b$ & $a$ & $c$ \\
$c$ & $e$ & $d$ & $d$ & $b$ & $a$ \\
$d$ & $a$ & $a$ & $e$ & $c$ & $b$
\end{tabular}

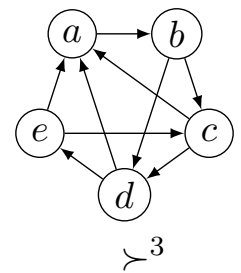

If an agent with preferences $\succsim_{1}^{3}: c, a, e, d, b$ joins the electorate, he changes $\succsim^{3}$ to $\succsim_{+1}^{3}$ where we have that $f\left(\succsim_{+1}^{3}\right)=\{b, d, e\}$. Consequently, nothing preferred to $\{b, d, e\}$ could have been selected by $f$ in $\succsim^{3}$ and we obtain $f\left(\succsim^{3}\right) \neq\{a\},\{c\},\{e\},\{a, c\},\{a, e\},\{c, e\}$, $\{d, e\},\{a, c, e\},\{a, d, e\},\{c, d, e\},\{a, c, d, e\},\{b, c, d, e\}, A$.

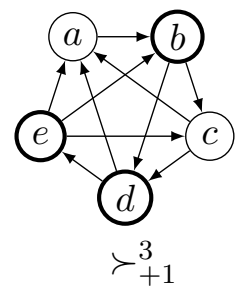

The same modification of $\succsim^{3}$ to $\succsim_{+1}^{3}$ can also be achieved by agents with preferences $\succsim_{2}^{3}: c, a, d, e, b$ and $\succsim_{3}^{3}: c, a, e, b, d$. This additionally gives $f\left(\succsim^{3}\right) \neq\{d\},\{a, d\},\{c, d\}$, $\{a, c, d\},\{a, b, d, e\}$ due to $\succsim_{2}^{3}$ and $f\left(\succsim^{3}\right) \neq\{b, e\},\{a, b, e\},\{b, c, e\},\{a, b, c, e\}$ because of $\succsim 3$.

We now let five different agents join the electorate, each of them will alter $\succsim^{3}$ to $\succsim_{+4}^{3}$ which is identical to $\succsim^{1}$. For this step of the proof suppose that $f\left(\succsim^{1}\right)$ is either $\{a, c, d, e\}$ or $A$. Adding an agent with preferences $\succsim_{4}^{3}: b, e, d, c, a$ then gives that $f$ cannot select $\{b\}$ or $\{b, e, d\}$ in $\succsim^{3}$. In a similar fashion, $\succsim_{5}^{3}: b, a, e, d, c$ excludes $\{a, b\}$ from the set of possible choices.

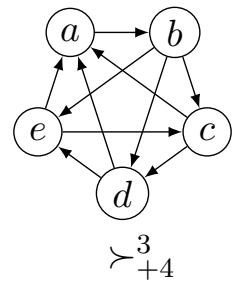
Next, if an agent with $\succsim_{6}^{3}: c, b, a, e, d$ joins the electorate, we obtain $f\left(\succsim^{3}\right) \neq\{b, c\},\{a, b, c\}$. An agent with $\succsim_{7}^{3}: d, b, a, e, c$ causes an identical change in the majority relation and gives that $f\left(\succsim^{3}\right)$ cannot be either $\{b, d\}$ or $\{a, b, d\}$. Finally, if an agent with preferences $\succsim_{8}^{3}: d, c, b, a, e$ joins the electorate, $\succsim^{3}$ is once more altered to $\succsim_{+4}^{3}$ and we deduce $f\left(\succsim^{3}\right) \neq\{b, c, d\},\{a, b, c, d\}$. 
To summarize this step, we have that if $f\left(\succsim_{+4}^{3}\right)=f\left(\succsim^{1}\right)$ is either $\{a, c, d, e\}$ or $A$, then $f\left(\succsim^{3}\right)=\emptyset$. Hence, $f\left(\succsim^{1}\right) \neq\{a, c, d, e\}, A$.

In the next step, we focus on $\succsim^{4}$ as depicted on the right. Just as before, we provide a preference profile inducing $\succsim^{4}$.

\begin{tabular}{llllll}
1 & 2 & 3 & 4 & 5 & 6 \\
\hline$a$ & $b$ & $b$ & $d$ & $e$ & $e$ \\
$b$ & $c$ & $c$ & $e$ & $a$ & $d$ \\
$c$ & $d$ & $d$ & $a$ & $b$ & $c$ \\
$d$ & $a$ & $e$ & $c$ & $c$ & $a$ \\
$e$ & $e$ & $a$ & $b$ & $d$ & $b$
\end{tabular}

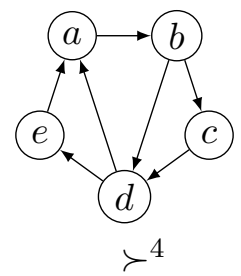

First, $\succsim^{4}$ can be altered to $\succsim_{+1}^{4}$ if an agent with preferences $\succsim_{1}^{4}: a, b, d, e, c$ joins the electorate. As we have $f\left(\succsim_{+1}^{4}\right)=\{a, b, d\}$, we obtain $f\left(\succsim^{4}\right) \neq\{a\}$ because otherwise joining would result in a strictly worse outcome for the corresponding agent.

A second agent with preferences $\succsim_{2}^{4}: b, d, e, c, a$ changes $\succsim^{4}$ to $\succsim_{+2}^{4}$, once again equal to $\succsim^{1}$. We now assume $f\left(\succsim^{1}\right)=\{a\}$. Given this presumption and that nothing preferred to $\{a\}$ may have been chosen in $\succsim^{4}$, we deduce that $f\left(\succsim^{4}\right) \neq X$ for all $X \in 2^{A} \backslash\{\emptyset,\{a\}\}$.

We hence have that if $f\left(\succsim_{+2}^{4}\right)=f\left(\succsim^{1}\right)=\{a\}$, then $f\left(\succsim^{4}\right)=\emptyset$. Thus, $f\left(\succsim^{1}\right) \neq\{a\}$.

Finally, consider $\succsim^{5}$ as given on the right together with a preference profile inducing it. Note that in $\succsim^{5}, c, d$, and $e$ are 'symmetric', i.e., as $f$ satisfies neutrality, $f$ has to either select all of $c, d, e$ or none of them.

\begin{tabular}{llllll}
1 & 2 & 3 & 4 & 5 & 6 \\
\hline$c$ & $c$ & $d$ & $d$ & $e$ & $e$ \\
$a$ & $b$ & $a$ & $b$ & $a$ & $b$ \\
$b$ & $d$ & $b$ & $e$ & $b$ & $c$ \\
$d$ & $e$ & $e$ & $c$ & $c$ & $d$ \\
$e$ & $a$ & $c$ & $a$ & $d$ & $a$
\end{tabular}
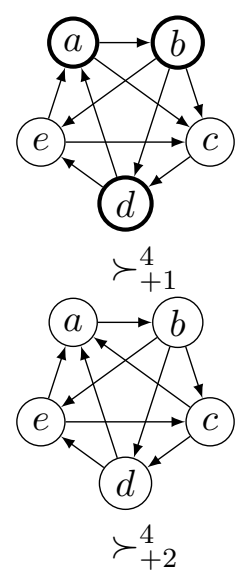

If an agent with preferences $\succsim_{1}^{5}: d, c, e, b, a$ joins the electorate, he changes $\succsim^{5}$ to $\succsim_{+1}^{5}$ where $b$ is a Condorcet winner and thus $f\left(\succsim_{+1}^{5}\right)=\{b\}$. Consequently, $f\left(\succsim^{5}\right) \neq\{c, d, e\},\{b, c, d, e\}$.

If on the other hand an agent with preferences $\succsim_{2}^{5}: a, b, d, c, e$ joins, $\succsim^{5}$ becomes $\succsim_{+2}^{5}$ which is equal to $\succsim^{1}$. Lastly supposing that $f\left(\succsim^{1}\right)$ is either $\{c, d, e\}$ or $\{b, c, d, e\}$, we can consequently exclude the remaining five choice possibilities from $\succsim^{5}: f\left(\succsim^{5}\right) \neq\{a\},\{b\},\{a, e\}$, $\{a, c, d, e\}^{12}, A$.

This step thus gives that if $f\left(\succsim_{+2}^{5}\right)=f\left(\succsim^{1}\right)$ equals $\{c, d, e\}$ or $\{b, c, d, e\}$, then $f\left(\succsim^{5}\right)=\emptyset$. We therefore have that

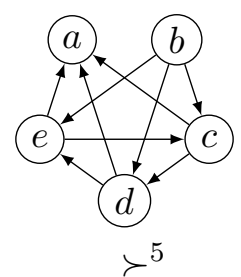
$f\left(\succsim^{1}\right) \neq\{c, d, e\},\{b, c, d, e\}$.

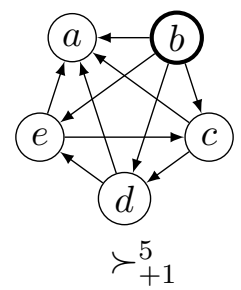

12. In case that $f\left(\succsim^{1}\right)=\{b, c, d, e\}$, an agent with preferences $\succsim_{3}^{5}: a, d, c, e, b$ has to join the electorate in order for us to be able to exclude $\{a, c, d, e\}$. 
To put it all together, we have that for $\succsim^{1}$, a majoritarian and Pareto-optimal SCF $f$ satisfying Fishburn-participation has to select either $\{a\},\{b\},\{a, b\},\{c, d, e\},\{a, c, d, e\}$, $\{b, c, d, e\}$, or $A$ due to neutrality. Yet, assuming $f$ selects $\{b\}$ or $\{a, b\}$, we have that $f\left(\succsim^{2}\right)=\emptyset$. If $f$ selects $\{a, c, d, e\}$ or $A$, we deduce that $f\left(\succsim^{3}\right)=\emptyset . f\left(\succsim^{1}\right)=\{a\}$ implies that $f\left(\succsim^{4}\right)=\emptyset$. Finally, if $f$ selects $\{c, d, e\}$ or $\{b, c, d, e\}$, we obtain $f\left(\succsim^{5}\right)=\emptyset$. We hence have that $f\left(\succsim^{1}\right)=\emptyset$, a contradiction. Thus, such an SCF $f$ cannot exist and the impossibility is proven.

The extension from $|A|=5$ to $|A| \geq 5$ works analogously to the extension in the proof of Theorem 1.

With respect to the number of agents observe that all relevant majority relations- $\succsim^{2}$, $\succsim^{3}, \succsim^{4}$, and $\succsim^{5}$-require an electorate of six agents to induce them. Together with the joining agent we obtain the threshold of $|N| \geq 7$ agents for this impossibility theorem to hold. ${ }^{13}$

\section{References}

Barberà, S., Bossert, W., \& Pattanaik, P. K. (2004). Ranking sets of objects. In Barberà, S., Hammond, P. J., \& Seidl, C. (Eds.), Handbook of Utility Theory, Vol. II, chap. 17, pp. 893-977. Kluwer Academic Publishers.

Biere, A. (2008). PicoSAT essentials. Journal on Satisfiability, Boolean Modeling and Computation, 4, 75-79.

Bordes, G. (1976). Consistency, rationality and collective choice. Review of Economic Studies, 43(3), 451-457.

Brandt, F. (2011). Minimal stable sets in tournaments. Journal of Economic Theory, $146(4), 1481-1499$.

Brandt, F. (2015). Set-monotonicity implies Kelly-strategyproofness. Social Choice and Welfare, 45(4), 793-804.

Brandt, F., \& Brill, M. (2011). Necessary and sufficient conditions for the strategyproofness of irresolute social choice functions. In Proceedings of the 13th Conference on Theoretical Aspects of Rationality and Knowledge (TARK), pp. 136-142. ACM Press.

Brandt, F., Brill, M., \& Harrenstein, P. (2016). Tournament solutions. In Brandt, F., Conitzer, V., Endriss, U., Lang, J., \& Procaccia, A. D. (Eds.), Handbook of Computational Social Choice, chap. 3. Cambridge University Press.

Brandt, F., Conitzer, V., \& Endriss, U. (2013). Computational social choice. In Weiß, G. (Ed.), Multiagent Systems (2nd edition)., chap. 6, pp. 213-283. MIT Press.

Brandt, F., Conitzer, V., Endriss, U., Lang, J., \& Procaccia, A. (Eds.). (2016). Handbook of Computational Social Choice. Cambridge University Press.

13. In principle, it is also possible to not have one agent join an electorate of size six, but have an agent with reversed preferences leave the electorate in order to achieve the same change in majority relations as well as consequences on the choice set. However, exhaustive search shows that for at least one of the majority graphs it is not possible to construct an electorate of size six such that one of the agents has exactly these reversed preferences. 
Brandt, F., \& Geist, C. (2016). Finding strategyproof social choice functions via SAT solving. Journal of Artificial Intelligence Research, 55, 565-602.

Brandt, F., Geist, C., \& Harrenstein, P. (2016). A note on the McKelvey uncovered set and Pareto optimality. Social Choice and Welfare, 46(1), 81-91.

Brandt, F., Geist, C., \& Peters, D. (2017). Optimal bounds for the no-show paradox via SAT solving. Mathematical Social Sciences, 90, 18-27. Special Issue in Honor of Hervé Moulin.

Brandt, F., Saile, C., \& Stricker, C. (2018). Voting with ties: Strong impossibilities via SAT solving. In Proceedings of the 17th International Conference on Autonomous Agents and Multiagent Systems (AAMAS), pp. 1285-1293. IFAAMAS.

Brandt, F., \& Seedig, H. G. (2016). On the discriminative power of tournament solutions. In Selected Papers of the International Conference on Operations Research, OR2014, Operations Research Proceedings, pp. 53-58. Springer-Verlag.

Chevaleyre, Y., Endriss, U., Lang, J., \& Maudet, N. (2007). A short introduction to computational social choice. In Proceedings of the 33rd Conference on Current Trends in Theory and Practice of Computer Science (SOFSEM), Vol. 4362 of Lecture Notes in Computer Science (LNCS), pp. 51-69. Springer-Verlag.

Ching, S., \& Zhou, L. (2002). Multi-valued strategy-proof social choice rules. Social Choice and Welfare, $19(3), 569-580$.

Debord, B. (1987). Caractérisation des matrices des préférences nettes et méthodes d'agrégation associées. Mathématiques et sciences humaines, 97, 5-17.

Dutta, B. (1990). On the tournament equilibrium set. Social Choice and Welfare, 7(4), $381-383$.

Dutta, B., \& Laslier, J.-F. (1999). Comparison functions and choice correspondences. Social Choice and Welfare, 16(4), 513-532.

Endriss, U. (Ed.). (2017). Trends in Computational Social Choice. AI Access.

Faliszewski, P., Hemaspaandra, E., \& Hemaspaandra, L. (2010). Using complexity to protect elections. Communications of the ACM, 53(11), 74-82.

Feldman, A. (1979). Manipulation and the Pareto rule. Journal of Economic Theory, 21, $473-482$.

Fishburn, P. C. (1972). Even-chance lotteries in social choice theory. Theory and Decision, 3(1), 18-40.

Fishburn, P. C. (1977). Condorcet social choice functions. SIAM Journal on Applied Mathematics, 33(3), 469-489.

Fishburn, P. C., \& Brams, S. J. (1983). Paradoxes of preferential voting. Mathematics Magazine, 56(4), 207-214.

Fisher, D. C., \& Reeves, R. B. (1995). Optimal strategies for random tournament games. Linear Algebra and its Applications, 217, 83-85.

Gärdenfors, P. (1976). Manipulation of social choice functions. Journal of Economic Theory, 13(2), 217-228. 
Gärdenfors, P. (1979). On definitions of manipulation of social choice functions. In Laffont, J. J. (Ed.), Aggregation and Revelation of Preferences. North-Holland.

Geist, C., \& Endriss, U. (2011). Automated search for impossibility theorems in social choice theory: Ranking sets of objects. Journal of Artificial Intelligence Research, 40, $143-174$.

Geist, C., \& Peters, D. (2017). Computer-aided methods for social choice theory. In Endriss, U. (Ed.), Trends in Computational Social Choice, chap. 13, pp. 249-267. AI Access.

Good, I. J. (1971). A note on Condorcet sets. Public Choice, 10(1), 97-101.

Holzman, R. (1988). To vote or not to vote: What is the quota?. Discrete Applied Mathematics, 22(2), 133-141.

Jimeno, J. L., Pérez, J., \& García, E. (2009). An extension of the Moulin No Show Paradox for voting correspondences. Social Choice and Welfare, 33(3), 343-459.

Kelly, J. S. (1977). Strategy-proofness and social choice functions without single-valuedness. Econometrica, 45(2), 439-446.

Laffond, G., Laslier, J.-F., \& Le Breton, M. (1993). The bipartisan set of a tournament game. Games and Economic Behavior, 5(1), 182-201.

Laslier, J.-F. (1997). Tournament Solutions and Majority Voting. Springer-Verlag.

Laslier, J.-F. (2000). Aggregation of preferences with a variable set of alternatives. Social Choice and Welfare, 17(2), 269-282.

McGarvey, D. C. (1953). A theorem on the construction of voting paradoxes. Econometrica, $21(4), 608-610$.

Moulin, H. (1988). Condorcet's principle implies the no show paradox. Journal of Economic Theory, 45(1), 53-64.

Pérez, J. (2001). The Strong No Show Paradoxes are a common flaw in Condorcet voting correspondences. Social Choice and Welfare, 18(3), 601-616.

Pérez, J., Jimeno, J. L., \& García, E. (2010). No show paradox in condorcet k-voting procedure. Group Decision and Negotiation, 21 (3), 291-303.

Pérez, J., Jimeno, J. L., \& García, E. (2015). No show paradox and the golden number in generalized Condorcet voting methods. Group Decision and Negotiation, 24, 497-513.

Sanver, M. R., \& Zwicker, W. S. (2012). Monotonicity properties and their adaption to irresolute social choice rules. Social Choice and Welfare, 39(2-3), 371-398.

Schwartz, T. (1986). The Logic of Collective Choice. Columbia University Press.

Scott, A., \& Fey, M. (2012). The minimal covering set in large tournaments. Social Choice and Welfare, 38(1), 1-9.

Sen, A. K. (1977). Social choice theory: A re-examination. Econometrica, 45(1), 53-89.

Smith, J. H. (1973). Aggregation of preferences with variable electorate. Econometrica, $41(6), 1027-1041$.

Tang, P., \& Lin, F. (2009). Computer-aided proofs of Arrow's and other impossibility theorems. Artificial Intelligence, 173(11), 1041-1053. 